\title{
The Use of Diode Laser in Treatment of Oral Submucous Fibrosis
}

\author{
${ }^{1}$ Ashish Soni, ${ }^{2}$ Sunil Sharma, ${ }^{3}$ Punit Chitlangia, ${ }^{4}$ Amit, ${ }^{5}$ Prateek Agarwal
}

\begin{abstract}
Oral submucous fibrosis (OSMF) is an established precancerous condition with increased prevalence in the Indian subcontinent. The treatment of OSMF is a challenging task for a clinician. Different authors have suggested variety of treatment modalities and have claimed success rates; still there is no universally acceptable protocol for the management of OSMF. In our case, transaction of bands was done by contact diode lasers (12 watt) under local anesthesia and it offered good results. Diode lasers offered excellent results and had many advantages over conventional surgical treatment.
\end{abstract}

Keywords: Diode laser, Local anesthesia, Oral submucous fibrosis.

How to cite this article: Soni $A$, Sharma $S$, Chitlangia $P$, Amit, Agarwal P. The Use of Diode Laser in Treatment of Oral Submucous Fibrosis. J Mahatma Gandhi Univ Med Sci Tech 2016;1(2):79-81.

\section{INTRODUCTION}

Oral submucous fibrosis (OSMF) is a chronic, debilitating disease characterized by juxtaepithelial fibrosis of the oral cavity. It is regarded as a precancerous and potentially malignant condition. ${ }^{1}$ Oral submucous fibrosis is a well known clinical entity since the time of "Sushruta" as 'Vidari'. ${ }^{2}$ The condition is thought to be multifactorial in origin with a high incidence in people who chew arecanut, and a significant malignant transformation rate (7-30\%) poses global problems for public health. The physical effects, which include a burning sensation in the mucosa and progressive trismus, can also have psychological and social implications for patients. ${ }^{1}$ The condition is found in 4/1,000 adults in rural India and as many as 5 million young Indians are suffering from this precancerous condition as a result of the increased popularity of the habit of chewing pan masala. Pan masala is a mixture of spices including, betel nuts, catechu, menthol, cardamom, lime, and others. ${ }^{3}$

\footnotetext{
${ }^{1}$ Postgraduate Student, ${ }^{2}$ Professor and Head, ${ }^{3}$ Reader ${ }^{4,5}$ Senior Lecturer

${ }^{1-5}$ Department of Oral and Maxillofacial Surgery, Mahatma Gandhi Dental College and Hospital, Jaipur, Rajasthan, India

Corresponding Author: Ashish Soni, 86/139, Pratap Nagar Sanganer, Jaipur, Rajasthan, India, e-mail: dr.ashishsoni29@ gmail.com
}

The most frequent location of OSMF is the buccal mucosa and the retromolar areas. It also commonly involves the soft palate, palatal fauces, uvula, tongue, and labial mucosa. Sometimes, it involves the floor of the mouth and gingival. ${ }^{4}$

Khanna and Andrade (1995) developed a group classification system for the surgical management of OSMF as follows: ${ }^{5}$

\begin{tabular}{ll}
\hline Group I & Interincisal distance greater than $35 \mathrm{~mm}$ \\
Group II & $\begin{array}{l}\text { Interincisal distance of } 26-35 \mathrm{~mm} \\
\text { Group III }\end{array}$ \\
$\begin{array}{l}\text { Interincisal distance of } 15-25 \mathrm{~mm} . \text { Fibrotic bands } \\
\text { are visible at the soft palate, pterygomandibular } \\
\text { raphe and anterior pillars of fauces }\end{array}$ \\
Group IVA & $\begin{array}{l}\text { Interincisal distance of less than } 15 \mathrm{~mm} \text { with } \\
\text { extensive fibrosis of oral mucosa all over }\end{array}$ \\
Group IVB & $\begin{array}{l}\text { Premalignant and malignant changes throughout } \\
\text { the mucosa (histologically) }\end{array}$ \\
\hline
\end{tabular}

There is no definite treatment for this condition. The various treatment modalities are mainly medical, surgical, or a combination of both. Conservative/ medical modality is the treatment of choice in patients with mild to moderate limitation in mouth opening. The nonsurgical line of treatment includes usage of gold, iodides, hyaluronidase, placental extract, and steroids like hydrocortisone and triamcinolone, vitamins, iron supplements. ${ }^{7}$ Whereas surgical treatment is the method of choice in patients with marked limitation of mouth opening or in patients not responding to the conservative management. ${ }^{8}$ Many surgical modalities like dermal graft, ${ }^{9}$ tongue flap, ${ }^{10}$ nasolabial flap, ${ }^{11}$ etc., are in vogue to cover the surgical defect created by transection of fibrous bands in oral mucosa.

Considering the limited success of the various treatment modalities and being aware of the fact that surgical treatment is the only palliative treatment having a fixed, limited target of relieving the trismus to enable the patient to overcome the morbidity, there is perpetual quest for the newer surgical protocols.

We report a case of oral submucous fibrosis treated with contact diode laser (12 watt) under local anesthesia for transection of fibrous bands. 


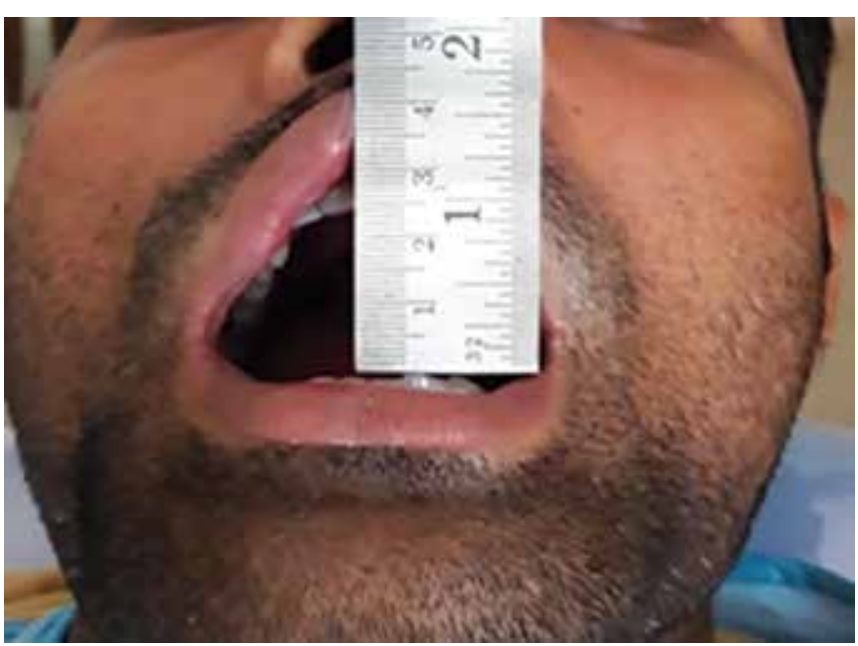

Fig. 1: Preoperative mouth opening

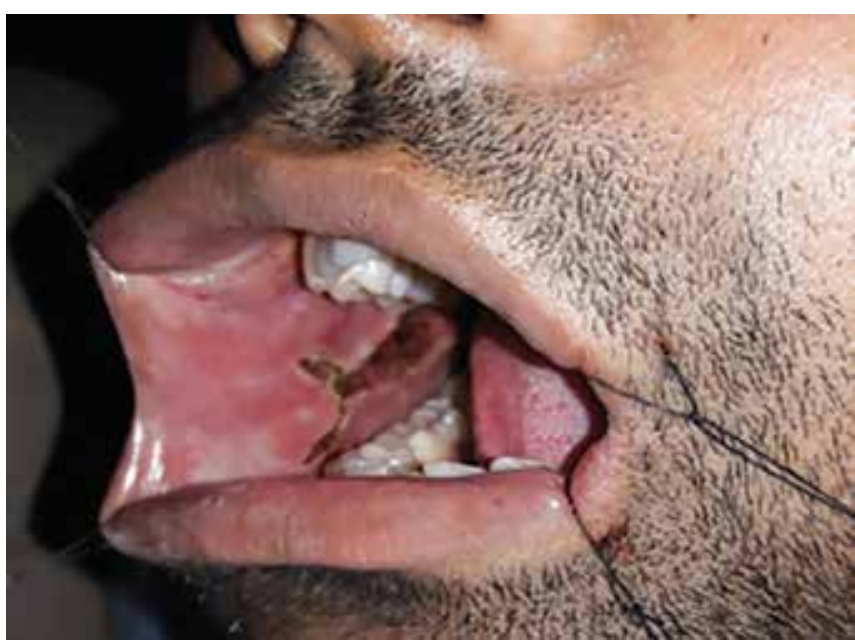

Fig. 3: Transection of fibrous bands by diode laser on right side

\section{CASE REPORT}

A 23-year-old male patient was reported in the department of oral and maxillofacial surgery with a chief complain of limited mouth opening since last 2 years and burning sensation on consumption of hot and spicy food. A detailed history was taken regarding tobacco chewing habit and dietary habit. It was revealed that patient consumed tobacco in the form of quid since last 5 years and also consumed excessive chillies. On examination fibrous bands were palpated on the right and left buccal mucosa extending up to the retromolar region. His preoperative mouth opening was recorded and was $24 \mathrm{~mm}$ (Fig. 1). Biopsy was taken under local anesthesia to confirm oral submucous fibrosis. After confirmation of biopsy report treatment was explained to the patient and transection of fibrous bands was done bilaterally by contact diode laser under local anesthesia and the raw area was not grafted and was allowed to epithelize on its own (Figs 2 and 3). Patient was kept on oral antibiotics and analgesics for 5 days and postoperative

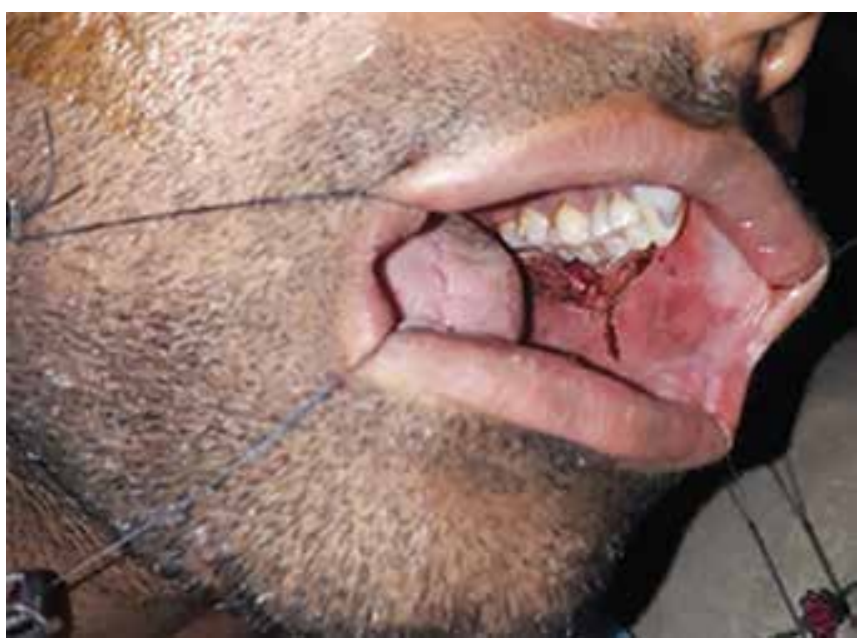

Fig. 2: Transection of fibrous bands by diode laser on left side

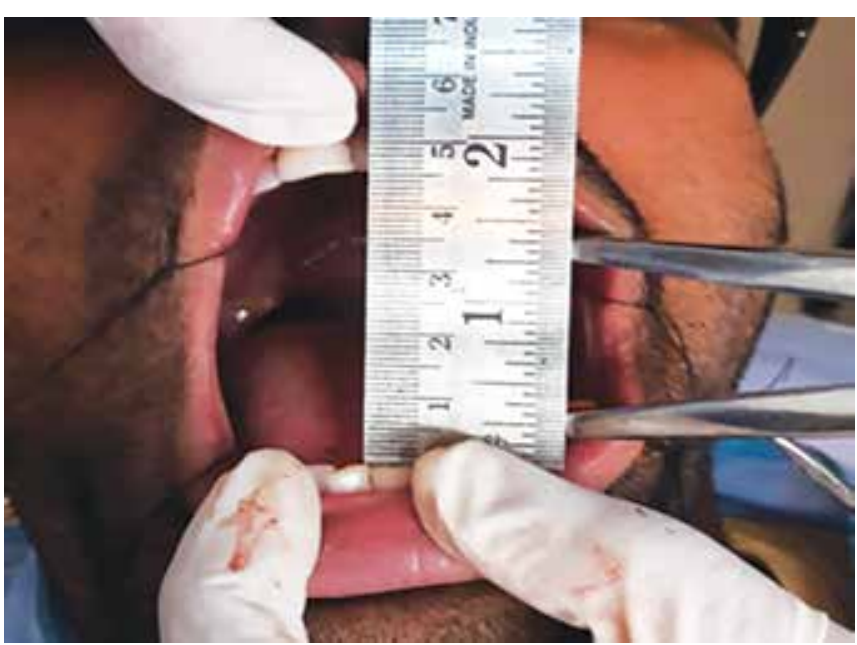

Fig. 4: Postoperative mouth opening

physiotherapy exercise was started after 48 hours, atleast for 7-8 times in a day at the interval of 1 hour. Patient was told to continue the exercise for a period of 1 year. The immediate postoperative mouth opening was $45 \mathrm{~mm}$ and subsequently was recorded also at the interval of 1 week, 1 and 6 months. Healing of the surgical site was completed in 4 weeks and also there was relief from burning sensation as the patient was kept on oral antioxidants for the same, and the final outcome was satisfactory.

\section{DISCUSSION}

Oral submucous fibrosis is an established precancerous condition with increased prevalence in the Indian subcontinent. An estimated 2.5 million people suffer from this disease in India. ${ }^{12}$ Prevalence rates of OSMF ranging from 0 to $17.6 \%$ have been reported in various population subsets. Betel nut chewing habit is the dominant etiological factor as majority of the patients are habituated to betel nut chewing in one form or the 
other. The onset of the disease is insidious and is often of 2 to 5 years duration. The most common initial symptom is burning sensation of the oral mucosa, aggravated by spicy food. Vesiculation, excessive salivation, ulceration, altered pigmentation, recurrent stomatitis, defective gustatory sensation, and dryness of the mouth have also been indicated as early symptoms. The treatment of oral submucous fibrosis is a challenging task for a clinician. All the documented treatment modalities, ranging from medicinal treatment to surgical treatment are purely symptomatic in nature and total cure of the disease is still elusive. Different authors have suggested variety of treatment modalities and claimed success rates; still there is no universally acceptable protocol for the management of OSMF. This is mainly due to the fact that the etiology of the disease is not fully understood and the disease is progressive in nature.

The basic aim of any type of treatment modality is to relieve the symptoms which include hampered function in the form of trismus, burning sensation in mouth, difficulty in mastication, deglutition and speech. Relieving such type of symptoms makes it a more difficult surgical problem. Surgeon should not only aim to relieve trismus but also should monitor for malignant transformation of this condition.

Recently, diode lasers have been used for excision of soft tissue lesions in oral cavity, also have been used for gingivectomies, curettage of the pockets, debridement of the root canals, for carrying out frenectomies, bleaching, etc. But, there are a few literatures available regarding their role in treatment of oral submucous fibrosis. In present case transection of bands was done by contact diode lasers (12 watt) under local anesthesia and it offered good results. There was significant improvement in mouth opening at the end of 6 months. The diode laser which was used in this case was of 7 watt and was kept on continuous mode. Diode laser offered several advantages over conventional surgical procedures like:

- The procedure done is a minor outpatient procedure under local anesthesia.

- Hemostatic nature of the surgery which allows surgery to be performed more precisely and accurately because of increased visibility and accessibility of the surgical site.

- Decreased postoperative pain and swelling.
- Causes a reduction in bacterial counts thereby promoting quicker, more predictable healing with minimal postoperative infection and inflammation.

- Leads to healing with minimal scarring.

Diode contact laser system can be used to rehabilitate grade III and early grade IV cases of OSMF. However, further more research is required for this system. A large sample size and long-term follow-up would give better insights for its use.

\section{CONCLUSION}

Till today, there is no modality which can be labeled as definitive treatment for oral submucous fibrosis. But, this protocol is worth of consideration and may give better results keeping in view the importance of postoperative mouth opening exercises and patient compliance.

\section{REFERENCES}

1. Arakeri G, Brennan PA. Oral submucous fibrosis: an overview of the aetiology, pathogenesis, classification, and principles of management. Br J Oral Maxillofac Surg 2013 Oct;51(7):587-593.

2. Mukherjee AL. Oral submucous fibrosis: A search for etiology. Ind J Otolaryngology 1972;24:11-15.

3. Ali M, Fareedi, Prasant MC, Patil A, Aher V. Oral submucous fibrosis: medical management. Global J Med Public Health 2012.Jan-Feb(1):12-18.

4. Ghom, Govindrao A. Oral premalignant lesions and conditions. Textbook of Oral Medicine. 2nd ed. Vol. 1. New Delhi Jaypee Brothers Medical Publishers, 2010 PP.194-225.

5. Khanna JN, Andrade NN. Oral submucous fibrosis: a new concept in surgical management. Report of 100 cases. Int J Oral Maxillofac Surg 1995;24:433-439.

6. More CB, Gupta S, Joshi J, Varma SN. Classification system for oral submucous fibrosis. J Indian Aca Oral Med Radiol 2012;24(1):24-29.

7. Marawetz G, Katsikers N, Weinberg S, Listrom R. Oral submucous fibrosis. J Oral Maxillofac Surg 1987;16:609-614.

8. Borle RM, Borle SR. Management of oral submucous fibrosis: a conservative approach. J Oral Maxillofac Surg 1991;49: 788-789.

9. Yen DJ. Surgical treatment of submucous fibrosis. J Oral Surg 1982 Sept:269-272.

10. Golhar S, Manohar MN, Narkhede S. Tongue flap in oral submucous fibrosis. Ind J Otolaryngol 1989;41:104-107.

11. Kavarana NM, Bhathena HM. Surgery for severe trismus in submucous fibrosis. British J of Plastic Surgery 1987;40:407409.

12. Aziz SR. Oral submucous fibrosis: case report and review of diagnosis and treatment. J Oral Maxillofac Surg 2008;66:23862389. 